\title{
THE X-RAY FLARE OF $\pi$ ' UMA : TEMPERATURE AND EMISSION MEASURE
}

M.Landini ${ }^{1}$ and B.C.Monsignori Fossi ${ }^{2}$

'(Department of Physics, University of Napoli, Italy)

${ }^{2}$ (Arceuri Astrophysical Observatory, Florence, Italy)

ABSTRACT. An X-ray flare from the star $\pi^{l}$ UMA has been observed by EXOSAT on January $3 I$. 19S4. The data were collected using the.Low Energy Experiment and the Medium Energy Experiment, sensitive to the spectral bands $0.04-2 \mathrm{Kev}$ and 1-20 Kev (Landini et al. 1986).

The temperature and emission measure variations during the flare are deduced under the isothermal assumption. Using a parametrized profile of the differential emission measure, wich is controlled by the conductive flux via the temperature gradient, the comparison with the observed data gives some insight on a "mean loop" model and its power supply.

\section{INTRODUCTION}

$\pi$ UMA (HD 72905) is a solar like star (G0 V) which has been observed with EXOSAT as part of a program devoled to the study of the X-Ray cmission from coronac of late type stars (Pallavicini et al. 1988). On January 31,1984 around 16 U.T. a large increase in the counting rate has been measured both by the low energy detector (LE) with the 3 Lexan filter and by the medium energy (ME) detector. A detailed discussion of the data and an isothermal interpretation of the observations have already been given (Landini et al. 1986). Here attention is put to estimate the time evolution of the temperature and cmission measere and to cvaluatc a "mean" model of the flare in $6 \mathrm{~mm}$ of a stationary loop.

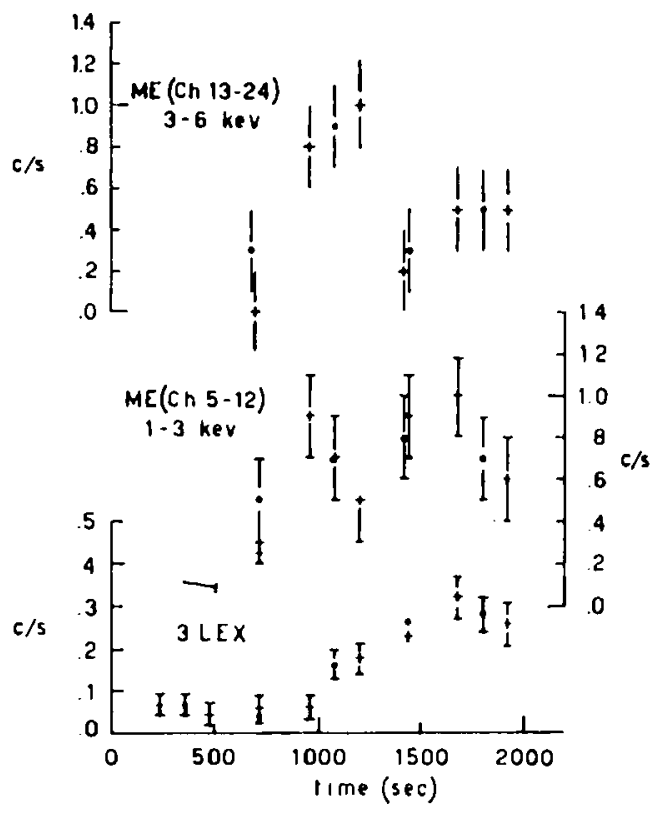

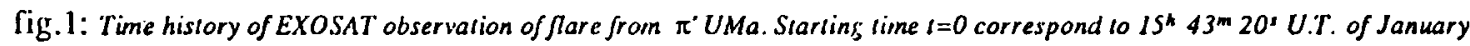
31, 1n84. Flux dala are counts/sec at the earth. Dots indicale daln averaged over 240 sec; cross: dala averaged over 360 sec. 


\section{THE TIME EVOLUTION OF TEMPERATURE AND EMISSION MEASURE.}

Due to the very low counting rate, ME data have been binned in two larger energy intervals concerning channels 5 to $12(1.1-3 \mathrm{Kcv})$ and 13 to 24 (3-6 Kev). Fig 1 shows the ligth curve of the event measured in the two ME bands and in the 3 Lexan filter $(0.04-2 \mathrm{Kev})$, after background subtraction. Data have been averaged over 240 sec. and 360 sec.; both are shown in the figure.

Since the counting statistics is rather poor and the data concern only three energy bands, the analysis has been developed assuming that the main contribution to the emission come from the quasi isothermall region which is the coronal part of the loop. The power emilted by unit emission measure from the flare plasma has been evalutated in the temperature interval $4.3<\log \mathrm{T}<8.0$ and filtered through the effective area of the detectors. By means of this information it is easy to evaluate the cmission measure which produces the observed count rate for any temperature from cach detector.

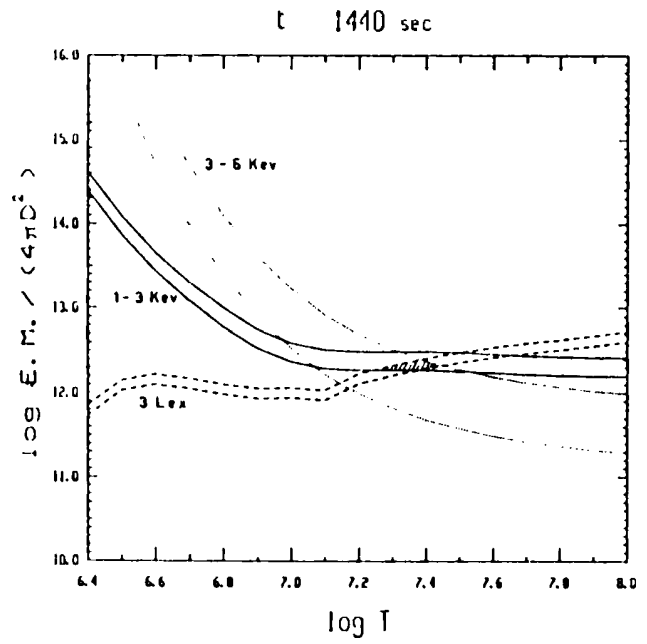

fig. 2: Isothermat analysis; the dashed region show's the temperature and emission measure which salisfy the signals \pm errors from all three dictclors. Any temperature larger thun $3.10^{7} \mathrm{~K}$ is a solution. Dala at time 1440 sec from the beginning of the event.

Fig. 2 show an example of the procedure applied to data at time $1440 \mathrm{sec}$. For each energy band, the signal \pm the error is used to generate the "strip "in the diagram which shows the emission measure versus temperature, compatible with the observation. If the three " strips "identify a common region in the diagram, this is the region of the solutions which satisfy all the three observations within the uncertaintics.

In fig. 3 the temperature and emission measure is plotted as a function of time. For comparison the dotled line shows an example of the time profile of the temperature for a solar flare observed by the SOLRAD satcllite using similar spectral bands (Landini and Monsignori Fossi 1979). A typical decay lime of about $600 \mathrm{sec}$. is obtained both in $\pi^{\prime}$ UMA and the Sun but the temperature of $\pi^{\prime}$ UMA is about twince the solar one. The emission measure remains almost costant all over the flare after a sharp increase in the carly phase. Also this time evolution is very similar to the solar case apart from the fact that the "lypical" solar emission measures are about $10^{49}-10^{50} \mathrm{~cm}^{-3}$, more than two orders of magnitude lower than the $\pi$ ' UMA flare.

Decaly limes of several hundred seconds require electron densities of the order of $10^{12} \mathrm{~cm}^{-3}$, if the radiation is the main cooling mechanism; this number density and the observed temperature claims for pressure very ne:ar to $10^{4}$ dyne $\mathrm{cm}^{-2}$. 


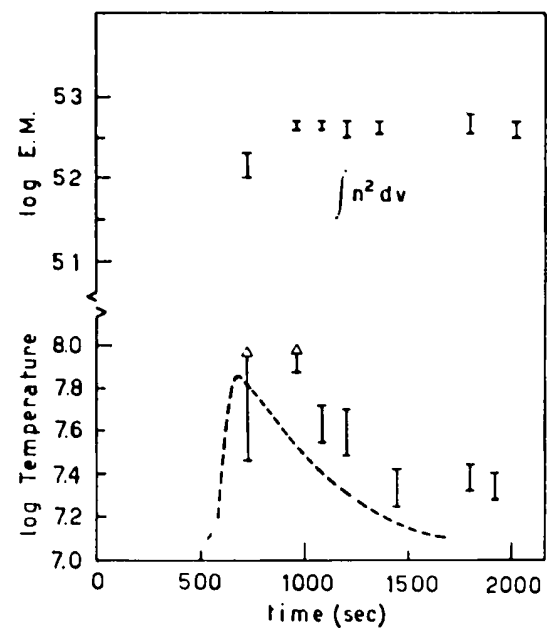

fig.3: Temperature and emission measure time evolution for the flare of $\pi^{\prime}$ UMa on January 31, 1984 at 16 U.T.; e.folding time decay is of the order of 600 sec; temperalure evolusion for a solar flare (Seplember 3, 1976) is shown for comparison.

\section{A "MEAN" LOOP MODEL OF THE FLARE.}

To have some insigth in the "mean" model for the loop, attention has been put on the part of the event where temperature seems to be stabilized ( $t \geq 900 \mathrm{sec}$ ). The count rates of ME bands and 3Lexan filter have been integrated and used to evaluate a multi-temperature loop model following a semi-cmpirical approach which proved to be useful for solar active regions (Monsignori Fossi and Landini,1988;.

The following parametric form is assumed for the conductive flux:

$$
F=F_{o} e^{-T_{0} / T}\left(T / T_{c}\right)^{\beta}\left[1-\left(T / T_{c}\right)^{0.5}\right]^{1 / 2}=F_{o} f\left(T, T_{c}, T_{0}, \beta\right)
$$

the differencial emission measure is therefore:

$$
S n^{2}(\mathrm{dh} / \mathrm{dT})=\left(\mathrm{p}_{0}^{2} \mathrm{~S} /\left(4 \mathrm{k}^{2} \mathrm{~F}_{0}\right)\right)\left(\mathrm{A} \mathrm{T}^{0 . .5} / \mathrm{f}\right) \quad\left(\mathrm{A}=10^{-6}\right)
$$

if costant pressure $p_{o}$ and cross section $S$ and completely ionized conduction are assumed. The parametrized differential cmission measure is used to generate simulated emission for proper selection of the parameters $T_{o}, \beta, T_{c}\left(\equiv\right.$ maximum temperature in the loop). A best $\chi^{2}$ fit procedure is used to estimate the parameters $T_{0}, \beta, T_{c}$ and $\left(p_{0}{ }^{2} S / F_{0}\right)$, which allow agreement between observed and predicted emission.

In order to have cnough data, the following channel binning has been used for the ME:

$\begin{array}{lll}0.89-1.81 \mathrm{keV} & 1.0710^{-1} \pm 0.9510^{-1} & \mathrm{c} / \mathrm{s} \\ 1.81-2.52 \mathrm{keV} & 2.3910^{-1} \pm 0.6910^{-1} & \mathrm{c} / \mathrm{s} \\ 2.52-3.49 \mathrm{keV} & 2.3710^{-1} \pm 0.7310^{-1} & \mathrm{c} / \mathrm{s} \\ 3.49-5.00 \mathrm{keV} & 2.0210^{-1} \pm 1.0210^{-1} & \mathrm{c} / \mathrm{s} \\ 5.00-6.31 \mathrm{keV} & 8.5510^{-2} \pm 8.0010^{-2} & \mathrm{c} / \mathrm{s} \\ 3 \text { Lexan } & 2.3010^{-1} \pm 0.2110^{-1} & \mathrm{c} / \mathrm{s}\end{array}$

The following results are obtained with a reduced $\chi^{2}$ less than 1: $\log T_{0}=5.6, \log T_{c}=7.5, \beta=0$.

From the parametric form of the conductive flux it is possible to evaluate in each point of the loop the conductive energy and the enthalpy flux for reasonable low value of the velocity $v_{0}$ at the base of the loop. By proper selection of the loop cross section $S$, the loop length and base velocity $v_{0}$, the 
energy bulance between radiative losses, conductive energy and divergence of the enthalpy flux may be investigated and indication of the distribution of the power supply along the loop may be obtained.

An example of this procedure is shown in fig 4 . In this case the cross section, the length and the base velocity have been selected in order to obtain energy balance for pressure slightly smaller tlan $10)^{4}$ dyne $\mathrm{cm}^{-2}$ as appears to be required by radiative decay times.

The temperature profile along the loop may be also obtained by integration of the conductive flux along the temperature once the length is known.

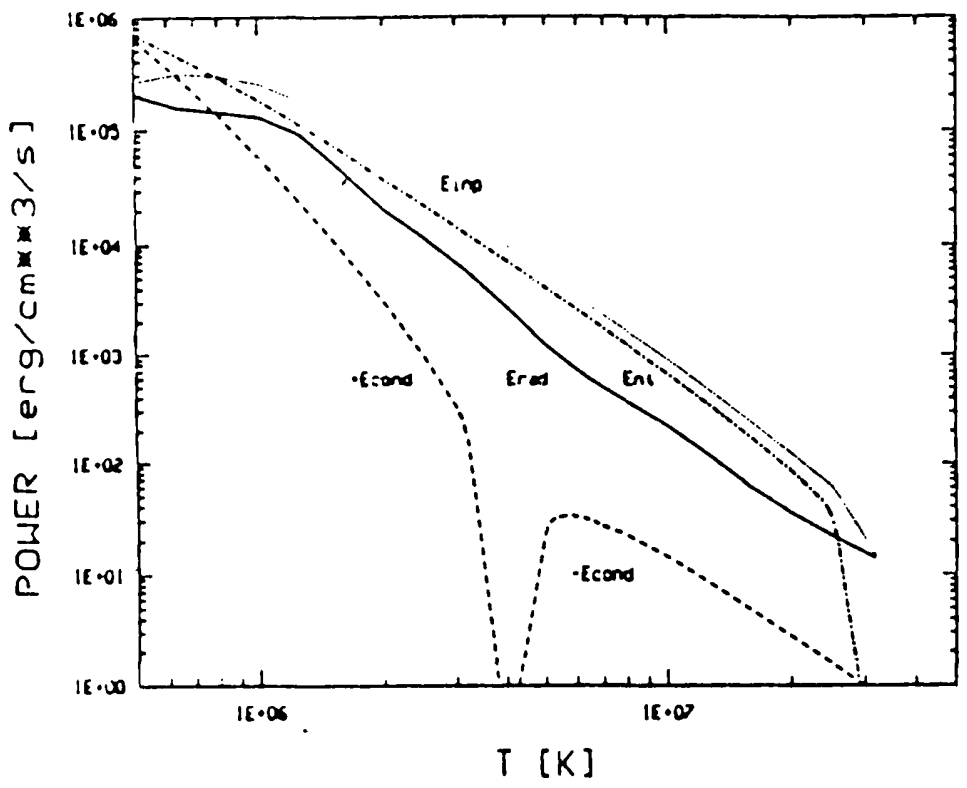

lig. 4: The encrgy balance for $L=10^{10} \mathrm{~cm}, S=1.710^{18} \mathrm{~cm}^{2}$ and base velocity vo $=3.10^{4} \mathrm{~cm} / \mathrm{sec}$. The coronal region of the loop must be hiated by an energy supply which decays according to a power law proportional to $\mathrm{T}^{-2.5}\left(n^{2.5}\right)$. The aspect ratio 1 loop ristius/scmi lingth/ is 0.07 . Valurs have been selected in order 10 oblain coronal number density compatibles with the ratiustive decay sime.

\section{CONCLUSIONS}

The lime evolution of temperature and emission measure for the flare of $\pi$ ' UMA has similar decaly times of those observed for the Sun $(-600 \mathrm{sec})$ but attains temperature twice higher and emission me:asures almost wo order of magnitude larger.

The use of a "semiempirical" model of the differential emission distributions inside a costant cross section and costint pressure loop. permits the evaluation of the loop length and temperature profile and allows to investigate the energy ballance.

\section{REFERENCES}

L.andini M.,Monsignori Fossi B.C.:1979, Astr. and Astroph.,72,171

L.andini M., Monsignori Fossi B.C.., Pallavicini R., Piro L.: 1986, Astr.and Astroph. 157, 217

Minnsignori Fossi B.C.., Landini M. : 1988, in "Aclivity in cool star envelopes", ed. (O.Havens el al. c(l.) 237

Pallivitini R., Monsignori Fossi B.C., Landini M. and Schmitl J.H.M.M.: 1988,Astr.and Astroph. 191, 109 